\title{
ATENÇÃO PRIMÁRIA À SAÚDE DO IDOSO: MODELO CONCEITUAL DE ENFERMAGEM*
}

\author{
Flavia Aparecida Dias ${ }^{1}$, Zenewton André da Silva Gama ${ }^{2}$, Darlene Mara dos Santos Tavares ${ }^{3}$
}

\begin{abstract}
RESUMO: O objetivo deste estudo foi elaborar um modelo conceitual sobre o papel do enfermeiro na atenção primária à saúde do idoso. Foi realizada revisão e análise documental de 40 documentos normativos brasileiros que regulam a atenção primária e/ou relacionados ao idoso, com coleta de dados entre abril e agosto de 2015. As informações foram consensuadas nos quatro metaparadigmas da enfermagem. O ser humano foi a pessoa de $>60$ anos; a saúde/doença: o conjunto de fatores físicos, psicológicos, sociais, econômicos, culturais e ambientais que permeiam o envelhecimento; e o ambiente: atenção primária, um conjunto de ações de saúde, no âmbito individual e coletivo, que abrange promoção e proteção da saúde, prevenção de agravos, diagnóstico, tratamento, reabilitação, redução de danos e manutenção da saúde; o enfermeiro, apresenta responsabilidade de gerência e cuidado à saúde. O modelo reflete as concepções específicas do idoso, saúde/doença, ambiente, bem como as atribuições do enfermeiro na atenção primária.
\end{abstract}

DESCRITORES: Idoso; Cuidados de enfermagem; Enfermagem; Enfermagem primária; Atenção primária à saúde.

\section{PRIMARY HEALTH CARE TO THE ELDERLY: A CONCEPTUAL MODEL OF NURSING}

ABSTRACT: The present study aimed to develop a conceptual model on the role of nurses in care to the elderly in primary health care services. Literature review and document analysis of 40 Brazilian normative documents that regulate primary care and/or care related to the elderly, and data was collected between April and August 2015. Consensus was obtained on information related to the four nursing meta paradigms. The human being was the person aged > 60 years; health/ disease: the set of physical, psychological, social, economic, cultural and environmental factors that pervade aging; and the environment: primary health care, a set of individual and collective health actions, covering health promotion and protection, disease prevention, diagnosis, treatment, rehabilitation, reduction of damage and health maintenance; the nurse, who is responsible for management and health care. The model reflects the concepts related to the elderly, health/disease environment, as well as nursing assignments in primary care.

DESCRIPTORS: Elderly; Nursing care; Nursing; Primary care nursing; Primary health care.

\section{ATENCIÓN BÁSICA A LA SALUD DEL ANCIANO: MODELO CONCEPTUAL DE ENFERMERÍA}

RESUMEN: Estudio cuyo intuito fue elaborar un modelo conceptual acerca del papel del enfermero en la atención básica a la salud del anciano. Se realizaron revisión y análisis documental de 40 documentos normativos brasileños que regulan la atención básica y/o relacionados al anciano. Se obtuvieron los datos entre abril y agosto de 2015. Se consensuaron las informaciones en los cuatro metaparadigmas de la enfermería. El ser humano fue la persona de $>60$ años; la salud/enfermedad: el conjunto de factores físicos, psicológicos, sociales, económicos, culturales y ambientales que están presentes durante el proceso de envejecimiento; y el ambiente: atención básica, un conjunto de acciones de salud, en ámbito individual y colectivo, que abarca promoción y protección de la salud, prevención de agravios, diagnóstico, tratamiento, rehabilitación, reducción de daños y manutención de la salud; el enfermero presenta responsabilidad de administración y cuidado a la salud. El modelo representa las concepciones específicas del anciano, salud/enfermedad, ambiente, así como las atribuciones del enfermero en la atención básica.

DESCRIPTORES: Anciano; Cuidados de enfermería; Enfermería; Enfermería básica; Atención básica a la salud. *Artigo extraído da tese intitulada: “Indicadores de processo da qualidade da assistência de enfermagem ao idoso na atenção
primária". Universidade Federal do Triângulo Mineiro, 2017.

${ }^{1}$ Enfermeira. Doutora em Atenção à Saúde. Universidade Federal do Triângulo Mineiro. Uberaba, MG, Brasil.

${ }^{2}$ Fisioterapeuta. Doutor em Exercício, Fisioterapia e Saúde. Docente da Universidade Federal do Rio Grande do Norte. Natal, $\mathrm{RN}$, Brasil.

${ }^{3}$ Enfermeira. Doutora em Enfermagem. Docente de Enfermagem da Universidade Federal do Triangulo Mineiro. Uberaba, MG, Brasil.

Autor Correspondente:

Darlene Mara dos Santos Tavares

Universidade Federal do Triangulo Mineiro

Av. Getúlio Guaritá, 159 - 38025-440 - Uberaba, MG, Brasil

E-mail: darlene.tavares@uftm.edu.br
Recebido: $12 / 06 / 2017$

Finalizado: $24 / 08 / 2017$ 


\section{○ INTRODUÇÃO}

A população idosa constitui-se na maior demanda aos serviços de saúde, com internações hospitalares mais frequentes e maior tempo de ocupação do leito. Repensar o planejamento do futuro da assistência à saúde torna-se necessário com foco no modelo de atenção à saúde, de modo a preparar-se para o grande crescimento da população idosa nas próximas décadas ${ }^{(1-2)}$.

Nesse contexto, destaca-se o papel da atenção primária como porta de entrada preferencial dos serviços de saúde. Neste espaço, todas as ações direcionadas à atenção à saúde do idoso são responsabilidade da equipe de saúde. Assim, é importante que a equipe seja formada por profissionais de diferentes áreas. Trata-se de um espaço privilegiado para o cuidado de enfermagem considerando o protagonismo do enfermeiro neste local, por suas responsabilidades específicas preconizadas pelo Ministério da Saúde quais sejam: planejar, gerenciar, coordenar, executar e avaliar a unidade de saúde, descritas também na Lei do Exercício Profissional ${ }^{(3-4)}$.

O processo de trabalho das equipes da atenção primária deve ser caracterizado, dentre outros, por ações que possam interferir no processo de saúde/doença da população, favorecendo o desenvolvimento da autonomia, individual e coletiva, bem como a qualidade de vida dos usuários. A programação das atividades deve atender aos critérios de problemas de saúde, ciclos de vida, sexo e morbidades ${ }^{(5)}$, evidenciando assim a relevância de conhecer o perfil populacional da clientela. Assim, torna-se necessário investigar a concepção de idoso, que pode ser distinta conforme as diversidades regionais.

Salienta-se que a atenção à saúde do idoso nesse espaço apresenta alguns desafios, como a necessidade de capacitação dos profissionais de saúde, ampliação da oferta de ações específicas direcionadas às necessidades do idoso e melhoria da qualidade da assistência.

Nesse sentido, considerando que no Brasil a atenção primária tem suas ações preconizadas pelo Ministério da Saúde, que deve ainda garantir fontes de recursos federais para compor o seu financiamento, é relevante conhecer as concepções relacionadas à atenção à saúde do idoso, bem como a assistência em saúde realizada pelo enfermeiro.

Para tanto, faz-se necessário a identificação de um modelo conceitual nesta temática, pois estas concepções norteiam as políticas públicas possibilitando visualizar o objeto de interesse e sua relação com questões relevantes direcionadas à saúde do idoso nesse cenário. Assim, pode-se contribuir para a melhoria da atenção prestada possibilitando que esta seja consoante ao que tem sido proposto pelo Ministério da Saúde. Baseando-se nestes antecedentes, o objetivo deste trabalho foi elaborar o modelo conceitual de enfermagem no cuidado a pessoas idosas na atenção primária à saúde.

\section{MÉTODOS}

Trata-se de uma revisão e análise de documentos normativos brasileiros que regulam a atenção primária e/ou relacionados ao idoso.

A estratégia de busca considerou como critérios de inclusão: documentos produzidos no Departamento de Atenção Básica do Ministério da Saúde (biblioteca oficial do site e material enviado em fase de publicação pela Coordenação de Saúde da Pessoa Idosa - COSAPI), Secretaria de Estado de Saúde Minas Gerais (biblioteca do site) e legislação nacional (portal da legislação do Governo Federal e legislação básica do Sistema Único de Saúde - portal da saúde); documentos relacionados ao idoso e/ou atenção primária e/ou atenção de enfermagem em saúde.

A busca dos referidos documentos foi realizada entre abril e agosto de 2015 sem delimitação do período de publicação, sendo incluídas todas disponíveis no período de 1994 (primeira publicação) a 2014 (última publicação).

$\mathrm{Na}$ biblioteca oficial do site do DAB/MS (http://dab.saude.gov.br/portaldab/biblioteca. php?conteudo=publicacoes), foram consultadas 265 publicações nas seções: políticas (4); cadernos (32); livros (32); cartilhas, guias e manuais (67); legislações da atenção básica em doenças crônicas (11) e 
SUS atenção básica (7), Melhor em Casa (89), Programa Nacional de Melhoria do Acesso e da Qualidade (12) e alimentação e nutrição (11); destaca-se que uma publicação se repetiu em duas seções, sendo selecionadas 31 .

Foi consultada também a seção de legislação, políticas e portarias nacionais de saúde e direitos dos idosos, indicados pelo Portal do Sistema de Indicadores de Saúde e Acompanhamento de Políticas do Idoso (SISAP-Idoso) do Ministério da Saúde (http://sisapidoso.icict.fiocruz.br/politicase-compromissos), totalizando 24 disponíveis sendo selecionadas seis, no entanto dessas, cinco já haviam sido incluídas em outros portais de busca e uma não contemplava o tema de estudo; no portal da legislação do governo federal, foi usado 'idoso' nos termos de busca e assunto, totalizando 44 documentos disponíveis sendo selecionado um documento.

No portal da saúde (http://portalsaude.saude.gov.br/index.php/links-de-interesse/89-cidadao/ legislacao/143-legislacao-basica-do-sus) foram encontrados 10 documentos na legislação básica do SUS sendo selecionado um. O material enviado via e-mail pela COSAPI, em fase de publicação, referese ao Manual para a utilização da Caderneta de Saúde da Pessoa Idosa, considerando que a versão disponível em formato eletrônico não estava atualizada.

Na biblioteca virtual da Secretaria de Estado de Saúde de Minas Gerais (http://www.saude.mg.gov.br/ sobre/publicacoes/linha-guia-e-manuais), foram analisadas as 12 linhas-guia disponíveis no site sendo incluídas neste estudo seis publicações.

Deste total (356) foram excluídos os documentos duplicados (6) e aqueles que não estavam relacionados aos quatro metaparadigmas de enfermagem (310). Deste modo, foram selecionados para análise 40 documentos ${ }^{(3,5-43)}$. A coleta de informações extraiu dados sobre a atenção ao idoso no contexto primário, bem como as atribuições do enfermeiro, sempre que disponíveis.

Foram realizadas leituras sucessivas de cada documento visando à extração de trechos para compor os metaparadigmas da enfermagem: idoso (ser humano), processo saúde/doença (saúde), atenção primária (ambiente) e enfermagem gerontológica (enfermagem). Após a apuração, o material foi organizado e analisado criticamente para a construção do modelo conceitual.

Para compor um modelo, deve-se identificar o conjunto de conceitos relativamente abstratos e gerais que abordam fenômenos de interesse central somado às suas proposições ${ }^{(44)}$. No campo da enfermagem, os conceitos relacionam-se aos quatro metaparadigmas da enfermagem: ser humano, saúde, ambiente e enfermagem ${ }^{(44-45)}$. Já as proposições podem ser classificadas como: não-relacionais (descrição ou definição de um conceito) ou relacionais (relação ou união entre dois ou mais conceitos) (44).

O modelo conceitual foi elaborado conforme os metaparadigmas, extraindo-se de cada documento os respectivos conceitos; as proposições foram analisadas e discutidas para a adequação do modelo. O painel de especialistas foi constituído pelos três coordenadores da pesquisa e uma pesquisadora ad hoc adicional; dentre os quatro analistas, três apresentavam doutorado e um mestrado sendo três da área da enfermagem e um da saúde coletiva. O modelo conceitual apresentado foi resultado dos documentos revisados e consenso dos pesquisadores envolvidos.

\section{RESULTADOS}

\section{Conceitos e proposições não-relacionais}

\section{Idoso}

Considera-se idoso, no Brasil, o indivíduo com 60 anos ou mais de idade ${ }^{(6-8,8)}$. Do ponto de vista social e psicológico, são mais heterogêneos que os jovens e vivenciam alterações estruturais e funcionais com o envelhecimento, que pode ser compreendido como um processo natural de diminuição progressiva da reserva funcional, denominado senescência ${ }^{(11,15,40)}$.

\section{Processo saúde doença}

O conceito de saúde para o idoso se traduz mais pela sua condição de autonomia e independência do 
que pela presença ou ausência de doença orgânica ${ }^{(11,16,40)}$. Deste modo, a saúde deve ser compreendida a partir de uma concepção ampliada, não sendo entendida apenas como ausência de doenças ${ }^{(11,16,31)}$. Trata-se de uma complexa produção social na qual os resultados na qualidade de vida se relacionam às decisões sobre os determinantes sociais ${ }^{(41)}$.

Em condições de sobrecarga como doenças, acidentes e estresse emocional, o processo de envelhecimento pode ocasionar uma condição patológica designada por senilidade, evidenciando a necessidade de assistência em saúde ${ }^{(15)}$.

As doenças podem ser desencadeadas considerando a repercussão das alterações decorrentes do processo fisiológico do envelhecimento, nos mecanismos homeostáticos do idoso e em sua resposta orgânica; assim, diminui sua capacidade de reserva, de defesa e de adaptação, tornando-o mais vulnerável aos estímulos traumáticos, infecciosos ou psicológicos ${ }^{(15)}$.

A presença de uma doença crônica não significa que o idoso não possa gerir sua própria vida e ser independente. A maioria dos idosos é capaz de decidir sobre seus interesses e se organiza sem necessidade de auxílio ${ }^{(8,40)}$. No entanto, as doenças crônicas e múltiplas geralmente associam-se à dependência desta população e perduram por vários anos, fazendo-se necessário acompanhamento médico e de equipes multidisciplinares permanentes ${ }^{(11,15,40)}$.

Salienta-se que a perda da funcionalidade se traduz em síndromes geriátricas e contribui para o comprometimento da qualidade de vida do idoso, de seus familiares e cuidadores $^{(40)}$. Soma-se a polifarmácia e fragilidades em diversas esferas e a mortalidade e morbidade por condições agudas, decorrentes de causas externas e agudizações de condições crônicas ${ }^{(31,40)}$.

\section{Atenção Primária}

No Brasil, os termos atenção primária e atenção básica são equivalentes ${ }^{(5)}$. Esta se caracteriza por um conjunto de ações de saúde, no âmbito individual e coletivo, que abrange a promoção e a proteção da saúde, a prevenção de agravos, o diagnóstico, o tratamento, a reabilitação, a redução de danos e a manutenção da saúde ${ }^{(5,40)}$. Assim, os seus elementos essenciais são: a educação sanitária, a imunização, a prevenção de endemias, o tratamento e prevenção das doenças, e a orientação para promoção de hábitos saudáveis ${ }^{(11)}$. O objetivo é desenvolver atenção integral que impacte na situação de saúde e autonomia e nos determinantes de saúde das coletividades ${ }^{(5)}$.

A atenção primária se constitui a principal porta de entrada sendo, ainda, o centro de comunicação da rede de atenção à saúde $e^{(5,8,11,40)}$. O foco das ações é a população adstrita, direcionando os profissionais à necessidade de conhecer as características do seu território. Este espaço de atenção apresenta mudanças permanentemente, no qual ocorrem relações sociais, de poder, de cooperação, de cooptação, de interação, dentre outras, onde a população reside, trabalha, constrói afetos, divertese, encontra-se e desencontra-se ${ }^{(31)}$.

O cuidado com o idoso envolve toda a rede de atenção, especialmente os serviços sociais e de saúde $^{(15)}$. Assim, a atenção primária ocorre na unidade de saúde, no domicílio, em locais do território como salões comunitários, escolas, creches, praças, e outros espaços que comportem a ação planejada $^{(5)}$; o idoso é acolhido pela equipe de saúde que deve captá-lo, acolhê-lo, desenvolver ações e avaliá-lo. É necessário o estabelecimento de vínculo entre o serviço e o idoso visando a garantia da assistência integral e contínua, englobando a família de forma humanizada, resolutiva, com qualidade e responsabilidade ${ }^{(11)}$.

Destaca-se que no domicílio pode ocorrer o cadastramento, a busca ativa, as ações de vigilância e de educação em saúde ${ }^{(31)}$; trata-se de um meio de vivência, interações, histórias e contextos que deve oferecer conforto e segurança ${ }^{(31,42)}$. No convívio do lar, o indivíduo recebe apoio familiar e do meio que o cerca. Deste modo, a família deve ser apoiada pela equipe nas mudanças de rotinas, normas e contextos ${ }^{(31)}$. Salienta-se o respeito no espaço domiciliar de modo a preservar os laços afetivos e fortalecer a autoestima; assim, pode-se contribuir para a construção de ambientes mais favoráveis à recuperação da saúde ${ }^{(31)}$.

Destaca-se entre as ações desenvolvidas na atenção primária a promoção da saúde no envelhecimento, que deve enfatizar o bom funcionamento físico, mental e social, a prevenção das 
morbidades e incapacidades, a melhoria da autonomia, a inclusão na família e na comunidade e aspectos relacionados à melhora da qualidade de vida, como o tratamento de cataratas, o investimento em cuidadores e espaços comunitários de convivência ${ }^{(11,27)}$.

\section{Enfermagem Gerontológica}

$\mathrm{Na}$ atenção ao idoso no contexto primário, são estabelecidas atribuições específicas ao enfermeiro e em conjunto com a equipe. Para classificação destas atribuições, consideraram-se as seguintes dimensões do trabalho do enfermeiro: administrar, assistir, ensinar, pesquisar e participar politicamente ${ }^{(46-47)}$, utilizadas na Estratégia Saúde da Família, porta de entrada da rede de atenção à saúde $^{(5,8,11,40)}$. No entanto, considerando que as atribuições obtidas nos documentos descrevem a prática no serviço de saúde, as dimensões possíveis nesta pesquisa são administrar/gerenciar e assistir/ cuidar, que têm sido as únicas duas dimensões referidas em pesquisas na atenção primária ${ }^{(48-49)}$.

São atribuições específicas do enfermeiro, na dimensão gerenciar: planejar, gerenciar, coordenar, executar e avaliar a unidade ${ }^{(3,12)}$; supervisionar e coordenar o trabalho dos agentes comunitários de saúde (ACS) e da equipe de enfermagem, ações de capacitação e facilitar a relação entre os profissionais de saúde e os $\operatorname{ACS}^{(3,5,12,15,21,23,39)}$. Na dimensão do cuidado, destacam-se: realizar procedimentos/ atendimentos e cuidados de enfermagem ${ }^{(3,5,34)}$; realizar consulta de enfermagem, com avaliações específicas $^{(3,5,10-15,18,25,27,32,35,39,41,43)}$; prescrever cuidados no domicílio ${ }^{(31)}$ e verificar a imunização do idoso ${ }^{(11)}$.

\section{Proposições relacionais}

- A maioria dos idosos apresenta boas condições físicas e sua saúde é boa, mas ao envelhecer perdem a capacidade de recuperar-se rápida e completamente das doenças ${ }^{(11)}$;

- Muitas medidas que afetam a saúde dos idosos transcendem o setor saúde, a saber: o estresse de agravos físicos, emocionais e sociais, como a viuvez ${ }^{(7,11,42)}$;

- A atenção integral e integrada à saúde do idoso deverá apresentar condições essenciais como infraestrutura física adequada, insumos e profissionais qualificados ${ }^{(16)}$;

- Na atenção primária, a avaliação diagnóstica do idoso deve considerar diversos fatores como o exame físico, os aspectos biológicos, psíquicos, funcionais, sociais, ambientais e a sua relação e dos familiares com o profissional de saúde, subentendendo-se que estes itens podem influenciar no seu envelhecimento e processo saúde/doença ${ }^{(15)}$;

- O envelhecimento e o processo saúde/doença possuem causas multifatoriais, sendo o envelhecimento influenciado pela discriminação e exclusão de gênero, etnia, racismo, hábitos de vida, condições socioeconômicas, acesso aos serviços públicos, região geográfica e local de moradia ${ }^{(15,40)}$. O processo saúde/doença está associado aos determinantes biológicos e socioculturais: as características pessoais, condições de saúde e relações formais e informais; as condições de vida e de trabalho, o acesso à alimentação, à educação, à produção cultural, ao emprego, à habitação, ao saneamento e aos serviços de saúde; e as condições socioeconômicas, culturais e ambientais dos indivíduos, famílias e redes sociais, e o desenvolvimento, a riqueza da localidade e sua distribuiç̧ão, e fatores políticos ${ }^{(11,16,31,41)}$;

- O idoso apresenta características peculiares quanto à apresentação, instalação e desfecho dos agravos em saúde, e maior vulnerabilidade a eventos adversos, necessitando de intervenções multidimensionais e multissetoriais ${ }^{(40)}$;

- $\quad$ O idoso utiliza mais os serviços de saúde, apresenta internações hospitalares mais frequentes e com maior tempo de ocupação do leito ${ }^{(11,40)}$;

- Os idosos diferem de acordo com a sua história de vida, seu grau de independência funcional e a demanda por serviços de saúde mais ou menos específicos ${ }^{(8,16)}$. Todos necessitam, contudo, de avaliação pautada no conhecimento do processo de envelhecimento e de suas peculiaridades adaptadas à realidade sociocultural em que estão inseridos ${ }^{(15-16)}$. Esta avaliação deve contemplar as dimensões social, clínica, mental e funcional, contribuindo na identificação das suas necessidades ${ }^{(40)}$.

Com base nos quatro metaparadigmas e proposições não-relacionais e relacionais, foi construído 
o modelo conceitual centrado na enfermagem, responsável por desenvolver ações relacionadas à gerência e ao cuidado na atenção primária, podendo assim influenciar no processo saúde/doença dos indivíduos, uma vez que neste espaço são desenvolvidas atividades relacionadas à promoção de saúde e prevenção de doenças, apresentado na Figura 1. Nesse contexto, evidencia-se que, além da atenção em saúde ofertada, as condições de saúde/doença do idoso podem ser influenciadas por diversos fatores pessoais, socioeconômicos, culturais, ambientais e políticos.

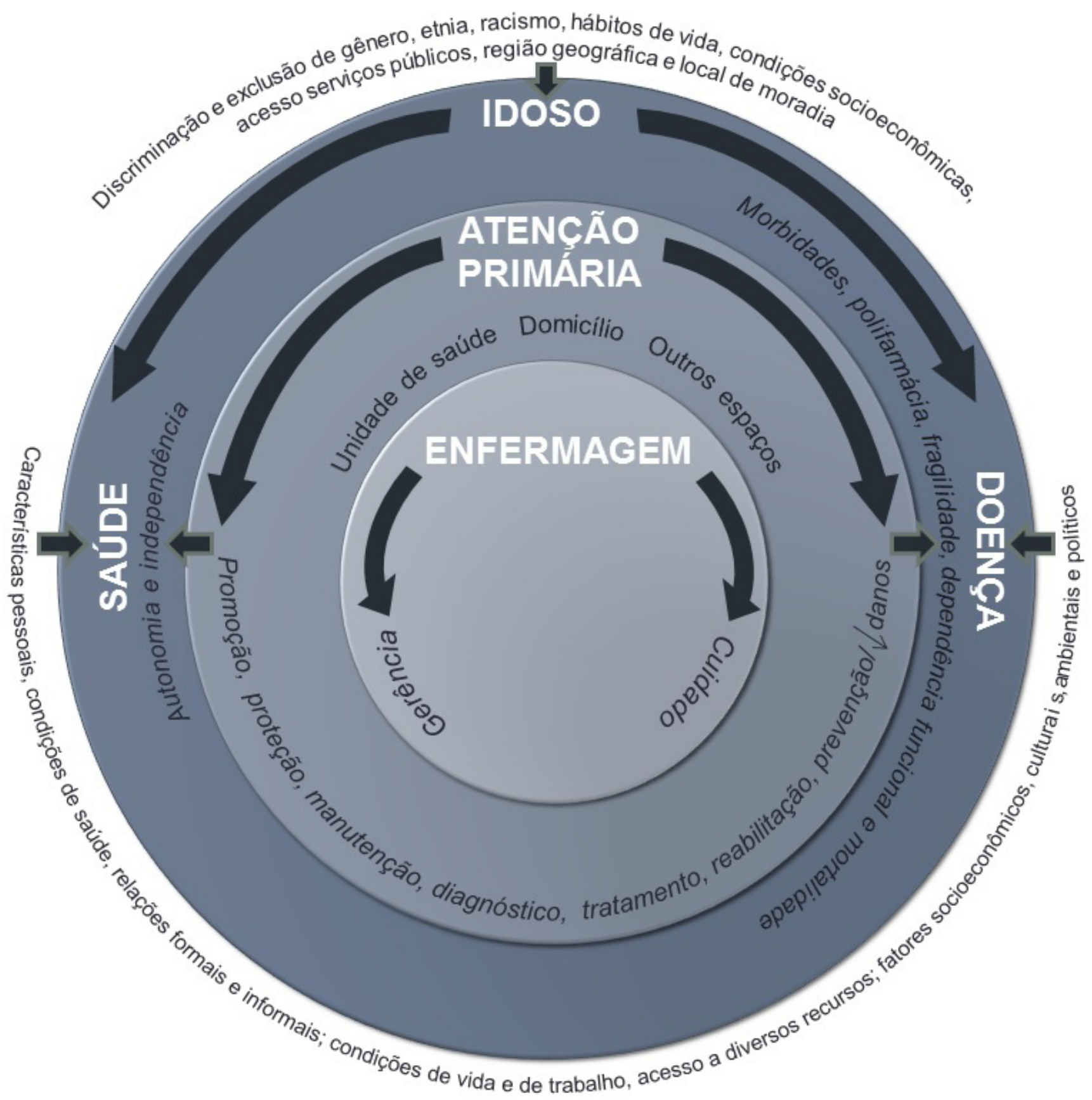

Figura 1 - Modelo conceitual para as dimensões do trabalho do enfermeiro na atenção primária ao idoso baseado nas publicações do Ministério da Saúde e Secretaria do Estado de Saúde de Minas Gerais. Uberaba, MG, Brasil, 2015

\section{DISCUSSÃO}

As concepções relacionadas ao processo saúde/doença entre os idosos evidenciam a necessidade do Sistema Único de Saúde (SUS) se reorganizar, diante da demanda crescente dos serviços de saúde 
para reabilitação física, mental e social do idoso. Nesse sentido, sugere que as ações em saúde estejam concentradas na prevenção de doenças, reabilitação dos agravos já instalados e promoção de saúde ${ }^{(1)}$.

Nesse contexto, destacam-se as recomendações de práticas preventivas para idosos, baseadas em evidências segundo o Programa de Actividades Preventivas y de Promoción de la Salud (PAPP) na atenção primária. Na última atualização do Grupo de Atividades Preventivas para Idosos do PAPPS, foram revisados os itens: exercício físico, quedas, medicação e detecção de fragilidade, e mantidas as recomendações anteriores relacionadas à demência e a déficits sensoriais ${ }^{(50)}$. Estas recomendações podem contribuir para maximizar a reflexão acerca das atividades preventivas de doenças nos serviços de atenção primária.

Quanto às ações desenvolvidas pelo enfermeiro neste espaço, a literatura destaca que frequentemente este profissional assume a função de coordenação ${ }^{(51-52)}$. No entanto, além de ações de gerência e planejamento, o enfermeiro desenvolve ações de cuidado direto e práticas educativas, que podem ser entendidas como estratégia de cuidado ampliado e integral na Estratégia Saúde da Família $(\mathrm{ESF})^{(53)}$.

O enfermeiro pode desenvolver na unidade de saúde atividades como os procedimentos específicos de sua categoria profissional na assistência, dentre eles: consulta de enfermagem, visita domiciliar, grupos, atividades de apoio e supervisão ao trabalho do agente comunitário de saúde (ACS) e do técnico ou auxiliar de enfermagem; soma-se a gerência da unidade de saúde ${ }^{(54)}$.

No que concerne à saúde do idoso, investigação no Paraná identificou a ausência de ações realizadas pelos enfermeiros; quando existentes, são de iniciativa da população e delegadas a outros profissionais de saúde. Observou-se que os enfermeiros realizam apenas cuidados curativos por relacionarem o processo de envelhecimento ao aspecto biológico ${ }^{(55)}$.

Inquérito realizado em Minas Gerais demonstrou que a assistência dos enfermeiros aos idosos não é sistematizada, ou seja, estes não recebem cuidados conforme suas necessidades em saúde ${ }^{(56)}$, fato também observado em outra investigação no Rio Grande do Sul(57). Já pesquisa realizada entre enfermeiros observou que as ações na ESF estão fundamentadas no modelo biomédico, não atendendo às recomendações nacionais dos documentos oficiais que regulamentam a sua implantação e funcionamento ${ }^{(54)}$.

Nesse sentido, faz-se necessário refletir sobre o papel da ESF, em especial do enfermeiro, na atenção ao idoso. Considerando o modelo conceitual deste estudo centrado na enfermagem, evidenciam-se as diversas responsabilidades em ações relacionadas à gerência e ao cuidado. Estas podem favorecer o processo de acompanhamento das condições de saúde do idoso com vistas à promoção de saúde e prevenção de doenças/agravos.

As limitações do estudo relacionam-se ao fato de se tratarem de documentos normativos, não sendo possível identificar os itens relacionados à definição e aos objetivos da enfermagem, para serem incorporados ao metaparadigma enfermagem gerontológica. Além disso, seria relevante a validação deste modelo por um painel maior de especialistas na área.

\section{- CONSIDERAÇÕES FINAIS}

A identificação de conceitos relacionados ao idoso na atenção primária pode contribuir para a reflexão acerca do trabalho do enfermeiro direcionado a este grupo populacional. Estudos posteriores estão sendo realizados no sentido de utilizar tal modelo para delinear indicadores que mensurem as atividades preconizadas direcionadas ao idoso no contexto primário. Assim, os gestores poderão utilizar tais dados para nortear o planejamento da atenção a saúde do idoso.

\section{REFERÊNCIAS}

1. Pilger C, Menon MU, Mathias TAF. Utilização dos serviços de saúde por idosos vivendo na comunidade. Rev. esc. enferm. USP. [Internet] 2013;47(1) [acesso em 12 jun 2017]. Disponível: http://dx.doi.org/10.1590/S008062342013000100027. 
2. Mendes ACG, de Sá DA, Miranda GMD, Lyra TM, Tavares RAW. Assistência pública de saúde no contexto da transição demográfica brasileira: exigências atuais e futuras. Cad. Saúde Pública. [Internet] 2012;28(5) [acesso em 12 jun 2017]. Disponível: http://dx.doi.org/10.1590/S0102-311X2012000500014.

3. Ministério da Saúde (BR). Guia prático do Programa Saúde da Família. [Internet] Brasília: Ministério da Saúde; 2001 [acesso em 04 mai 2015]. Disponível: http://dab.saude.gov.br/portaldab/biblioteca.php?conteudo=publicacoes/ guia_pratico_saude_familia.

4. Conselho Federal de Enfermagem. Lei n. 7.498, de 25 de junho de 1986. Dispõe sobre a regulamentação do exercício da enfermagem, e dá outras providências. Diário Oficial da União, 26 jun 1986.

5. Ministério da Saúde (BR). Portaria n. 2.488, de 21 de outubro de 2011. Aprova a Política Nacional de Atenção Básica, estabelecendo a revisão de diretrizes e normas para a organização da Atenção Básica, para a Estratégia Saúde da Família (ESF) e o Programa de Agentes Comunitários de Saúde (PACS). Diário Oficial da União, 24 Out 2011. Seção 1.

6. Ministério da Saúde (BR). Lei n. 8.842, de 4 de janeiro de 1994. Dispõe sobre a Política Nacional do Idoso, cria o Conselho Nacional do Idoso e dá outras providências. Diário Oficial da União, 05 Jan 1994. Seção 1.

7. Ministério da Saúde (BR). Secretaria de Políticas de Saúde. Departamento de Atenção Básica. Instabilidade postural e quedas. [Internet] Brasília: Ministério da Saúde; 1999 [acesso em 25 mai 2015]. Disponível: http:// bvsms.saude.gov.br/bvs/publicacoes/atencao_saude_idoso_cab4.pdf.

8. Ministério da Saúde (BR). Secretaria de Assistência à Saúde. Redes Estaduais de Atenção à Saúde do Idoso: guia operacional e portarias relacionadas. [Internet] Brasília: Ministério da Saúde; 2002 [acesso em 04 mai 2015 ]. Disponível: http://bvsms.saude.gov.br/bvs/publicacoes/redes_estaduais.pdf.

9. Presidência da República (BR). Lei n. 10.741, de $1^{\circ}$ de outubro de 2003. Dispõe sobre o Estatuto do Idoso e dá outras providências. Diário Oficial da União, 02 Out 2003. Seção 1.

10. Ministério da Saúde (BR). Secretaria de Atenção à Saúde. Departamento de Atenção Básica. Avaliação para melhoria da qualidade da estratégia saúde da família. [Internet] Brasília: Ministério da Saúde; 2005 [acesso em 03 ago 2015]. Disponível: http://189.28.128.100/dab/docs/publicacoes/geral/doc_tec_amq_portugues.pdf.

11. Minas Gerais (BR). Secretaria de Estado de Saúde. Atenção a saúde do idoso. [Internet] Belo Horizonte: SAS/MG; 2006 [acesso em 04 mai 2015]. Disponível: http://www.saude.mg.gov.br/images/documentos/ LinhaGuiaSaudeldoso.pdf.

12. Minas Gerais (BR). Secretaria de Estado de Saúde. Atenção à Saúde do Adulto - tuberculose. [Internet] Belo Horizonte: SAS/MG; 2006 [acesso em 04 mai 2015]. Disponível: http://www.saude.mg.gov.br/images/documentos/ LinhaGuiaTuberculose2006.pdf.

13. Minas Gerais (BR). Secretaria de Estado de Saúde. Atenção a saúde do adulto - HIV/AIDS. [Internet] Belo Horizonte: SAS/MG; 2006 [acesso em 04 mai 2015]. Disponível: http://www.saude.mg.gov.br/images/documentos/ LinhaGuiaHIVAIDS.pdf.

14. Minas Gerais (BR). Secretaria de Estado de Saúde. Atenção a saúde do adulto - hanseníase. [Internet] Belo Horizonte: SAS/MG; 2006 [acesso em 04 mai 2015]. Disponível: http://www.saude.mg.gov.br/images/documentos/ LinhaGuiaHanseniase.pdf.

15. Ministério da Saúde (BR). Secretaria de Atenção à Saúde. Departamento de Atenção Básica. Envelhecimento e saúde da pessoa idosa. [Internet] Brasília: Ministério da Saúde; 2006 [acesso em 04 mai 2015]. Disponível: http:// bvsms.saude.gov.br/bvs/publicacoes/envelhecimento_saude_pessoa_idosa_n19.pdf.

16. Ministério da Saúde (BR). Portaria n. 2.528, de 19 de outubro de 2006. Aprova a Política Nacional de Saúde da Pessoa Idosa. Diário Oficial da União, 20 Out 2006. Seção 1.

17. Ministério da Saúde (BR). Portaria n. 399, de 22 de fevereiro de 2006. Divulga o Pacto pela Saúde 2006 Consolidação do SUS e aprova as Diretrizes Operacionais do Referido Pacto. Diário Oficial da União, 23 Fev 2006. Seção 1.

18. Ministério da Saúde (BR). Secretaria de Atenção à Saúde. Departamento de Atenção Básica. Prevenção clínica de doenças cardiovasculares, cerebrovasculares e renais. [Internet] Brasília: Ministério da Saúde; 2006 [acesso 
em 10 ago 2015]. Disponível: http://dab.saude.gov.br/portaldab/biblioteca.php?conteudo=publicacoes/cab14.

19. Ministério da Saúde (BR). Secretaria de Atenção à Saúde. Departamento de Atenção Básica. Obesidade. [Internet] Brasília: Ministério da Saúde; 2006 [acesso em 10 ago 2015]. Disponível: http://189.28.128.100/dab/docs/ publicacoes/cadernos_ab/abcad12.pdf.

20. Ministério da Saúde (BR). Secretaria de Atenção à Saúde. Departamento de Atenção Básica. Hipertensão arterial sistêmica para o Sistema Único de Saúde. [Internet] Brasília: Ministério da Saúde; 2006 [acesso em 10 ago 2015]. Disponível: http://189.28.128.100/dab/docs/publicacoes/cadernos_ab/abcad15.pdf.

21. Ministério da Saúde (BR). Secretaria de Atenção à Saúde. Departamento de Atenção Básica. Diabetes mellitus. [Internet] Brasília: Ministério da Saúde; 2006 [acesso em 10 ago 2015]. Disponível: http://189.28.128.100/dab/docs/ publicacoes/cadernos_ab/abcad16.pdf.

22. Ministério da Saúde (BR). Secretaria de Atenção à Saúde. Departamento de Atenção Básica. Protocolos do Sistema de Vigilância Alimentar e Nutricional - SISVAN na assistência à saúde. [Internet] Brasília: Ministério da Saúde; 2008 [acesso em 26 ago 2015]. Disponível: http://189.28.128.100/dab/docs/portaldab/publicacoes/ protocolo_sisvan.pdf.

23. Ministério da Saúde (BR). Secretaria de Atenção à Saúde. Departamento de Atenção Básica. Vigilância em Saúde: Dengue, Esquistossomose, Hanseníase, Malária, Tracoma e Tuberculose. [Internet] Brasília: Ministério da Saúde; 2008 [acesso em 10 ago 2015]. Disponível: http://189.28.128.100/dab/docs/publicacoes/cadernos_ab/ abcad21.pdf.

24. Ministério da Saúde (BR). Secretaria de Atenção à Saúde. Departamento de Atenção Básica. Caderno de AutoAvaliação n.5: Equipe Saúde da Família - Parte 2. ( $3^{a}$ ed.). [Internet] Brasília: Ministério da Saúde; 2009 [acesso em 04 mai 2015]. Disponível: http://www.capital.ms.gov.br/sesau/wp-content/uploads/sites/30/2017/03/20110331090406. pdf.

25. Ministério da Saúde (BR). Secretaria de Atenção à Saúde. Departamento de Atenção Básica. Vigilância em saúde: zoonoses. [Internet] Brasília: Ministério da Saúde; 2009 [acesso em 10 ago 2015]. Disponível: http://189.28.128.100/ dab/docs/publicacoes/cadernos_ab/abcad22.pdf.

26. Minas Gerais (BR). Secretaria de Estado de Saúde. Linha Guia de Atenção à Saúde - dengue. [Internet] Belo Horizonte: SAS/MG; 2009 [acesso em 04 mai 2015]. Disponível: http://www.fhemig.mg.gov.br/images/stories/ protocolos_clinicos/linha_guia_de_atencao_a_saude_dengue_ses_mg.pdf.

27. Ministério da Saúde (BR). Secretaria de Atenção à Saúde. Departamento de Atenção Básica. Rastreamento. [Internet] Brasília: Ministério da Saúde; 2010 [acesso em 07 ago 2015]. Disponível: http://bvsms.saude.gov.br/bvs/ publicacoes/caderno_atencao_primaria_29_rastreamento.pdf.

28. Ministério da Saúde (BR). Secretaria de Atenção à Saúde. Departamento de Atenção Básica. Doenças respiratórias crônicas. [Internet] Brasília: Ministério da Saúde; 2010 [acesso em 10 ago 2015]. Disponível: http://189.28.128.100/dab/docs/publicacoes/cadernos_ab/abcad25.pdf.

29. Ministério da Saúde (BR). Secretaria de Atenção à Saúde. Departamento de Atenção Básica. Saúde sexual e saúde reprodutiva. [Internet] Brasília: Ministério da Saúde; 2010 [acesso em 07 ago 2015]. Disponível: http://189.28.128.100/dab/docs/publicacoes/cadernos_ab/abcad26.pdf.

30. Ministério da Saúde (BR). Secretaria de Atenção à Saúde. Departamento de Atenção Básica. Acolhimento à demanda espontânea. [Internet] Brasília: Ministério da Saúde; 2011 [acesso em 07 ago 2015]. Disponível: http://189.28.128.100/dab/docs/publicacoes/geral/miolo_CAP_28.pdf.

31. Ministério da Saúde (BR). Secretaria de Atenção a Saúde. Departamento de Atenção Básica. Caderno de atenção domiciliar. Vol.1. [Internet] Brasília: Ministério da Saúde; 2012 [acesso em 04 mai 2015]. Disponível: http://189.28.128.100/dab/docs/publicacoes/geral/cad_vol1.pdf.

32. Ministério da Saúde (BR). Secretaria de Atenção à Saúde. Departamento de Atenção Básica. Autoavaliação para a Melhoria do Acesso e da Qualidade da Atenção Básica: AMAQ. [Internet] Brasília: Ministério da Saúde; 2012 [acesso em 03 ago 2015]. Disponível: http://189.28.128.100/dab/docs/sistemas/pmaq/amaq.pdf.

33. Ministério da Saúde (BR). Secretaria de Atenção à Saúde. Departamento de Atenção Básica. Acolhimento à demanda espontânea: queixas mais comuns na Atenção Básica. [Internet] Brasília: Ministério da Saúde; 2012 
[acesso em 10 ago 2015]. Disponível: http://189.28.128.100/dab/docs/publicacoes/cadernos_ab/caderno_28.pdf.

34. Ministério da Saúde (BR). Secretaria de Atenção a Saúde. Departamento de Atenção Básica. Caderno de atenção domiciliar. Vol.2. [Internet] Brasília: Ministério da Saúde; 2013 [acesso em 04 mai 2015]. Disponível: http://189.28.128.100/dab/docs/portaldab/publicacoes/cad_vol2.pdf.

35. Ministério da Saúde (BR). Secretaria de Atenção à Saúde. Departamento de Atenção Básica. Controle dos cânceres do colo do útero e da mama. [Internet] Brasília: Ministério da Saúde; 2013 [acesso em 26 ago 2015]. Disponível: http://189.28.128.100/dab/docs/portaldab/publicacoes/cab13.pdf.

36. Ministério da Saúde (BR). Secretaria de Atenção à Saúde. Departamento de Atenção Básica. Estratégias para o cuidado da pessoa com doença crônica: hipertensão arterial sistêmica. [Internet] Brasília: Ministério da Saúde; 2013 [acesso em 26 ago 2015]. Disponível: http://189.28.128.100/dab/docs/portaldab/publicacoes/caderno_37.pdf.

37. Ministério da Saúde (BR). Secretaria de Atenção à Saúde. Departamento de Atenção Básica. Estratégias para o cuidado da pessoa com doença crônica: diabetes mellitus. [Internet] Brasília: Ministério da Saúde; 2013 [acesso em 26 ago 2015]. Disponível: http://189.28.128.100/dab/docs/portaldab/publicacoes/caderno_36.pdf.

38. Ministério da Saúde (BR). Secretaria de Atenção à Saúde. Departamento de Atenção Básica. Instrumento de Avaliação Externa para as Equipes de Atenção Básica - saúde da família e equipe parametrizada. [Internet] Brasília: Ministério da Saúde; 2013 [acesso em 03 ago 2015]. Disponível: http://189.28.128.100/dab/docs/portaldab/ documentos/instrumento_ae_sfp.pdf.

39. Minas Gerais (BR). Secretaria de Estado de Saúde. Linha-guia de hipertensão arterial sistêmica, diabetes mellitus e doença renal crônica. [Internet] Belo Horizonte: SAS/MG; 2013 [acesso em 04 mai 2015]. Disponível: http://www.saude.mg.gov.br/images/documentos/guia_de_hipertensao.pdf.

40. Ministério da Saúde (BR). Secretaria de Atenção à Saúde. Departamento de Atenção Especializada e Temática. Coordenação Saúde da Pessoa Idosa. Diretrizes para o cuidado das pessoas idosas no SUS: proposta de modelo de atenção integral à saúde da pessoa idosa. [Internet] Brasília: Ministério da Saúde; 2014 [acesso em 21 ago 2015]. Disponível: http://portalsaude.saude.gov.br/images/pdf/2015/setembro/01/Diretrizesaten----opessoaidosa.pdf.

41. Ministério da Saúde (BR). Secretaria de Atenção à Saúde. Departamento de Atenção Básica. Estratégias para o cuidado da pessoa com doença crônica. [Internet] Brasília: Ministério da Saúde; 2014 [acesso em 18 ago 2015]. Disponível: http://bvsms.saude.gov.br/bvs/publicacoes/estrategias_cuidado_pessoa_doenca_cronica_cab35. pdf.

42. Ministério da Saúde (BR). Manual para a utilização da Caderneta de Saúde da Pessoa Idosa. [Internet] Brasília: Ministério da Saúde; 2014 [acesso em 18 ago 2015]. Disponível: https://www.icict.fiocruz.br/sites/www.icict. fiocruz.br/files/201402061526_manualcadernetadoidoso.pdf.

43. Ministério da Saúde (BR). Secretaria de Atenção à Saúde. Departamento de Atenção Básica. Estratégias para o cuidado da pessoa com doença crônica: obesidade. [Internet] Brasília: Ministério da Saúde; 2014 [acesso em 18 ago 2015]. Disponível: http://bvsms.saude.gov.br/bvs/publicacoes/estrategias_cuidado_doenca_cronica_ obesidade_cab38.pdf.

44. Fawcett J, DeSanto-Madeya S. Contemporary Nursing Knowledge: Analysis and Evaluation of Nursing Models and Theories. EUA: F.A. Davies Company; 2013.

45. Hickman JS. Introdução à teoria da enfermagem. In: George JB e colaboradores. Teorias de enfermagem - os fundamentos à prática profissional. $4^{\mathrm{a}}$ ed. Trad. de Ana Maria Vasconcellos Thorell. Porto Alegre: Artmed, 2000. p.11-20.

46. Sanna MC. Os processos de trabalho em Enfermagem. Rev. bras. enferm. [Internet] 2007;60(2) [acesso em 12 jun 2017]. Disponível: http://dx.doi.org/10.1590/S0034-71672007000200018.

47. de Paula M, Peres AM, Bernardino E, Eduardo EA, Sade PMC, Larocca LM. Características do processo de trabalho do enfermeiro da Estratégia de Saúde da Família. Reme, Rev. Min. Enferm. [Internet] 2014;18(2) [acesso em 12 jun 2017]. Disponível: http://www.dx.doi.org/10.5935/1415-2762.20140034.

48. Matumoto S, Fortuna CM, Kawata LS, Mishima SM, Pereira MJB. Nurses' clinical practice in primary care: a process under construction. Revista Latino-Am. Enfermagem. [Internet] 2011;19(1) [acesso em 12 jun 2017]. Disponível: http://dx.doi.org/10.1590/S0104-11692011000100017. 
49. Guedes MVC, Silva LF, Macêdo NR, Brasil RWN, Linhares MSC, Francelino MFF, et al. Sobral-Ceará: cenário de desenvolvimento da CIPESQ-Brasil. In: Chianca TCM, Antunes MJM, organizadores. A Classificação Internacional das Práticas de Enfermagem em Saúde Coletiva: CIPESC. Brasília (DF): ABEn; 1999. p.109-23.

50. Lesende IM, Hidalgo JDLT, Iturbe AG, Alonso MCH, Diéz JMB, Herreros YH. Actividades preventivas en los mayores. Atención Primária. 2014;46(4):75-81.

51. Melo RC, Machado ME. Coordenação de unidades de saúde da família por enfermeiros: desafios e potencialidades. Rev. Gaúcha Enferm. [Internet] 2013;34(4) [acesso em 08 ago 2017]. Disponível: http://dx.doi. org/10.1590/S1983-14472013000400008.

52. Spagnuolo RS, Juliani CMCM, Spiri WC, Bocchi SCM, Martins STF. O enfermeiro e a estratégia saúde da família: desafios em coordenar a equipe multiprofissional. Cienc Cuid Saude. [Internet] 2012;11(2) [acesso em 08 ago 2017]. Disponível: http://www.revenf.bvs.br/pdf/ccs/v11n2/02.pdf.

53. da Silva LMS, Fernandes MC, Mendes EP, Evangelista NC, Torres RAM. Trabalho interdisciplinar na estratégia saúde da família: enfoque nas ações de cuidado e gerência. Rev. enferm. UERJ. [Internet] 2012;20(6) [acesso em 08 ago 2017]. Disponível: http://www.e- http://www.e-publicacoes.uerj.br/index.php/enfermagemuerj/article/ view/6024.

54. Pinheiro GML, Alvarez AM, de Pires DEP. A configuração do trabalho da enfermeira na atenção ao idoso na Estratégia de Saúde da Família. Ciênc. saúde coletiva. [Internet] 2012;17(8) [acesso em 01 mar 2017]. Disponível: http://dx.doi.org/10.1590/S1413-81232012000800021.

55. Pilger C, Dias JF, Kanawava C, Baratieri T, Carreira L. Compreensão sobre o envelhecimento e ações desenvolvidas pelo enfermeiro na atenção primária à saúde. Cienc. enferm. [Internet] 2013;19(1) [acesso em 12 jun 2017]. Disponível: http://dx.doi.org/10.4067/S0717-95532013000100006.

56. Resende JO, Silva FMR, Assunção RS, Quadros KAN. Assistência do enfermeiro ao idoso na estratégia saúde da família. Rev. enferm. Cent.-Oeste Min. [Internet] 2015;5(3) [acesso em 08 ago 2017]. Disponível: http://www. seer.ufsj.edu.br/index.php/recom/article/view/880/935.

57. Alberti GF, Espíndola RB, Carvalho SORM. The approach to the elderly in the family health strategy and the implications for nursing practice. Rev. pesqui. cuid. fundam. (Online). [Internet] 2014;6(2) [acesso em 08 ago 2017]. Disponível: http://www.seer.unirio.br/index.php/cuidadofundamental/article/view/3118/pdf_1266. 